Case Report

\title{
PROLONGED PYREXIA WITH ACUTE RENAL INSULT - UNRAVELING THE PUZZE AND SOLVING THE ENIGMA
}

\author{
Raghava Sharma \\ Professor, Department of M edicine, K. S. Hegde M edical Academy, Nitte University, M angalore - 575 018, \\ Karnataka, India. \\ Correspondence \\ Raghava Sharma \\ Professor, Department of Medicine, K. S. Hegde Medical Academy, Nitte University, Deralakatte, M angalore - 575018 , \\ Karnataka, India. E-mail : rrsharma1967@yahoo.com
}

\begin{abstract}
:
A middle aged man with prolonged pyrexia was referred to us with a diagnosis of FUO (Fever of unknown origin). He was evaluated by various investigations and a diagnosis of Tuberculosis was established. On anti tubercular treatment (ATT) he developed complication of acute renal injury -non oliguric renal failure, from which he recovered fully after the puzzle was successfully solved \& managed accordingly. Even though he was proved to have rifampicin induced acute intersticial nephritis(AIN) by biopsy, he had varied \& unconventional presentation like without oliguria, without peripheral blood eosinophilia, and more so particularly on the maiden administration of rifampicin. Thus our case highlights the importance of quickly establishing the cause for FUO, and also need for greater vigilance on part of physicians to solve unconventional presentations of complications arising out of treatment.
\end{abstract}

Keywords: FUO, Fever of unknown origin, Tuberculosis, Rifampicin, Acute renal insult, acute intersticial nephritis

\section{Introduction:}

FUO (Fever of unknown origin) is a common condition encountered in clinical practice particularly at tertiary care medical college hospitals. In the present case, we encountered a common disorder (tuberculosis) presenting uncommonly (as FUO) \& developing an uncommon complication on treatment (drug induced renal injury) which also presented with varied/ uncommon presentation (which has been fully discussed under uniqueness of the case). Thus this case highlights the importance of being vigilant about uncommon $\&$ varied presentation of the case \& complications and the role of multiple investigations to prove the diagnosis, and the difficulties in solving the enigma \& puzzle when an uncommon complication with uncommon \& varied presentation sets in while on treatment.

\begin{tabular}{|c|}
\hline Access this article online \\
\hline Quick Response Code \\
\hline
\end{tabular}

\section{Case Report:}

A 55 year old male was referred to our tertiary care medical college hospital with a diagnosis of FUO (Fever of unknown origin) of one month duration, and with no other history of any previous diseases nor any comorbid conditions. Investigations done for evaluation of M alaria, Tuberculosis, and Enteric fever were negative in the previous hospital.

On arrival at our hospital, his medical history was reviewed. He had history of low grade intermittent fever, mostly towards evenings. There were no significant cardio vascular symptoms or symptoms related to any other system. He had loss of appetite with some weight loss over the previous month. For his fever he had received treatment with antibiotics on several occasions.

General examination revealed no significant findings. BP was 130/80 mmHg, Pulse rate was 98/min and respiratory rate was $26 / \mathrm{min}$. On respiratory system examination he was found to have Bronchial breath sound with increased VF/VR over the right infraclavicular and mammary areas. Examination of other systems did not reveal any positive findings.

His investigation reports were as follows: $\mathrm{Hb}-11.6 \mathrm{~g} \%$; $\mathrm{TC}$ : 22,000 cells/cumm, DC: N- 85\%, E- 1\%, L-12\%, M- 2\%; ESR97mm/hr; RBS: 141mg\%; Urea: 36; Creatinine: $1.5 \mathrm{mg} \%$; LFT- WNL; Chest X-ray: Right upper lobe consolidation with infiltrates over left lower lobe areas (Fig 7). Sputum culture: No growth. Sputum cytology was negative for 
malignant cells. Of the three sputum samples for AFB, two samples were positive. This was later confirmed by positive BAL (Broncho alveolar lavage) samples for AFB. Bronchoscopy demonstrated inflammatory picture of right upper lobe bronchial tree mucosa (Fig 1-4). AFB serology (IgM ) for Tuberculosis was negative and Sputum PCR for TB was also negative.

To begin with based on preliminary evaluation he was initiated on injectable broad spectrum antibiotic for right upper lobe pneumonia. Once Pulmonary TB was confirmed, ATT with CAT-1 treatment was started. With this treatment patient was showing signs of recovery and was discharged at request on ATT.

Within a week of discharge patient came back with worsening of his previous symptoms associated with nausea and vomiting. On examination he had no significant general examination findings. His BP was 130/90 mmhg, Respiratory rate- 22/min, Temperature- 99F, Pulse rate$104 /$ min. His investigation reports were as follows: $\mathrm{Hb}$ 11.5g\%; TC- 24,000cells/cumm; LFT- WNL; Urea- 75mg\%; Creatinine- $3.5 \mathrm{mg} \%$; Urine analysis- Albumin +, WBC 68/HPF, RBC 2-4/HPF, No crystals/casts.

As our patient had non oliguric renal failure, nephrology opinion was sought and further investigations were done accordingly (ASO titer: Negative, C3 complement level: Normal, Abdominal Ultrasound: Normal, 24 hr. urine protein: $1 \mathrm{~g} / \mathrm{d}$ ). Based on the above reports, in view of rapid deterioration in renal functions, a working diagnosis of Rapidly progressive glomerulonephritis (RPGN)/ Drug induced Acute intersticial nephritis(AIN ) was made. Kidney biopsy was planned and performed. Biopsy report was suggestive of "Acute intersticialnephritis" (Fig 5, 6). ATT was continued without Rifampicin. NSAID given for Pleuritic chest pain was stopped. Prednisolone $30 \mathrm{mg} /$ day along with Amlodipine $10 \mathrm{mg} /$ day for high BP was initiated. With this his renal functions improved and he was discharged, two weeks after his second admission, with a diagnosis of Pulmonary Tuberculosis and Drug induced (? Rifampicin/ NSAID) intersticial nephritis.
During his immediate follow-up visit an attempt was made to reintroduce Rifampicin. On doing so, renal functions deteriorated. Hence it became clear that patient had Rifampicin induced acute intersticial nephritis thus conclusively proving the etiology of intersticial nephritis. He was advised to continue with ATT without Rifampicin and Levofloxacin $750 \mathrm{mg} /$ day was added instead. Prednisolone was tapered over the next few weeks and stopped.

He was reviewed further regularly on a monthly basis and he responded well to the treatment. At the end of completion of his ATT course (after 6 months) the investigation reports were Urea- $24 \mathrm{mg} \%$, Creatinine$1 \mathrm{mg} \%$, ESR- $12 \mathrm{~mm} / \mathrm{hr}$, and Chest X-ray-Normal.

\section{Discussion :}

FUO (Fever of unknown origin) was defined by Petersdorf and Beeson in 1961 as temperature $>38.3 \mathrm{C}(101 \mathrm{~F})$, lasting for more than 3 weeks and failure to reach a diagnosis even after 1 week of inpatient investigations. A subsequent definition in 2003 stated that failure to get a diagnosis after 3 days of inpatient investigations or 3 outpatient visits should qualify for the term FUO.

Tuberculosis (TB) is one of the world's most wide spread and deadly diseases infecting $1 / 3$ of world's population and is caused by acid fast bacillus M ycobacterium tuberculosis. TB is an important cause of FUO. The most common site of the disease is lungs and most common extra pulmonary sites are lymph nodes, pleura, bones, and joints. TB is a major cause of mortality and morbidity worldwide but chiefly in resource limited settings.

A study on the frequency and pattern of adverse reactions to essential Antitubercular drugs revealed an incidence of $16.9 \%$ and adverse drug reactions were generally caused by an individual drug. In $5.6 \%$ of cases the adverse reactions were intractable which made the specific drug to be discontinued ${ }^{1}$.

"Rifampicin" is one of the most effective bactericidal drugs in the anti tubercular treatment (ATT) regimen. Serious adverse drug reactions due to Rifampicin are uncommon. 


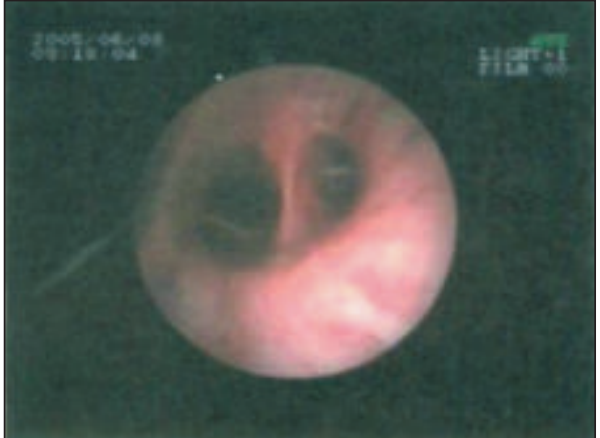

Fig 1

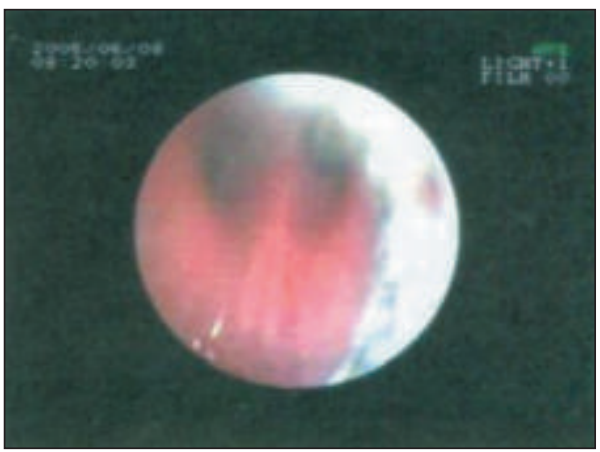

Figs 1 to 4:

Fig 3

RUL Bronchial tree mucosa inflamed with copious secretions. Rest of Bronchial tree normal.

Micro photographs of Kidney Biopsy:

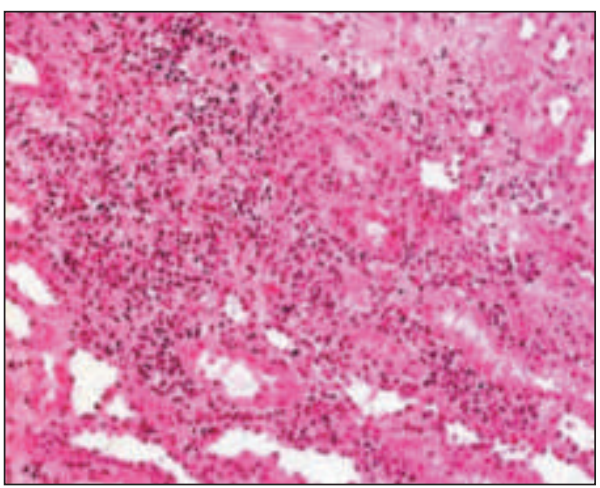

Fig 5

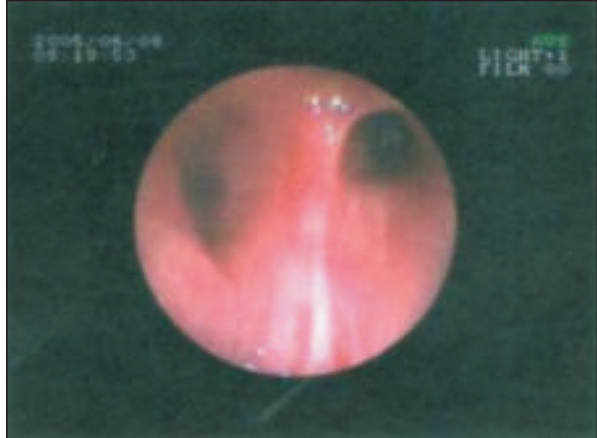

Fig 2

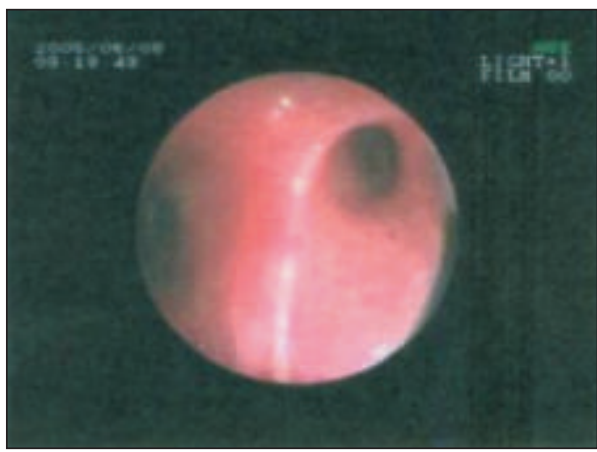

Fig 4

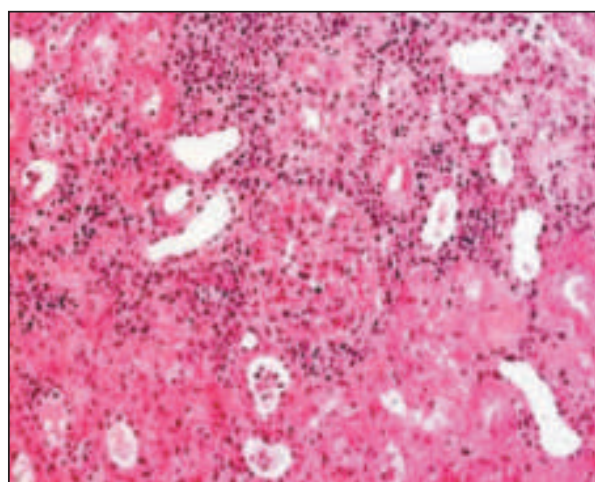

Fig 6

Fig $5 \& 6$ : Intersticial and tubular infiltrations by inflammatory cells suggestive of Acute intersticial nephritis.

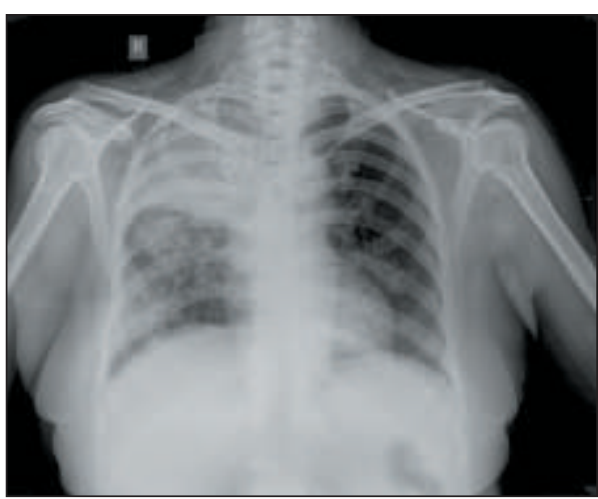

Fig 7: Chest X ray : Right upper lobe consolidation with infiltrations over Left lower lobe areas.
However Rifampicin may induce major adverse drug reactions like Thrombocytopaenia ${ }^{2}$, Acute renal failure ${ }^{3}$, and Toxic Hepatitis. Acute intersticial nephritis (AIN) and/or acute tubular necrosis (ATN) is the most frequent renal leision in Rifampicin induced acute renal failure ${ }^{4}$.

In humans, most drug induced AIN probably involves cell mediated immunity as renal biopsies usually do not disclose any immune deposits and intersticial infiltrates usually contain a considerable percentage of $T$ cells- 
Inflamatory cells, M ononuclear cells, Leucocytes including Eosinophils 5 .

AIN is one of the major causes of reversible acute kidney injury. Early diagnosis of AIN, early identification of causative drug and its withdrawal is the main stay of treatment. Additionally use of high dose glucocorticoids helps to eradicate inflamatory infiltrates more rapidly $\&$ completely and may thus minimize sussequent chronic damage ${ }^{6}$.

Rifampicin induced AIN is usually reversible if detected early and treated appropriately.

\section{Uniqueness of Present Case:}

A. Rifampicin induced Acute renal failure -AIN has been reported among the TB patients following retreatment (at an interval ranging from 5 months to 11 years) with an intermittent Rifampicin regimen especially in those who have been previously treated with daily Rifampicin containing regimens. The mechanism postulated for this phenomenon is that, during the gap in the treatment or during intermittent dose regimen large quantities of antibodies accumulate and later intense immune reaction develops to subsequent Rifampicin administration further leading to renal injury ${ }^{7}$. BUT IN OUR CASE IT WAS THE MAIDEN ADM INISTRATION OF RIFAM PICIN (ATT) THAT INDUCED RENAL INJURY AND PROVED TO BE ACUTE INTERSTICIAL NEPHRITIS- AIN BY BIOPSY.

B. "Peripheral blood eosinophilia" is reported to be an

\section{References:}

1. M ishin vlu, Vasileva IA, Makieva VG, Kuzmina NV, Prikazchikova AV, Khoroshutina VV. Probl Tuberk Bolezn LegK. 2003; (7): 24-9

2. Rajendraprasad, Mukerjee Pk. Rifampicin induced thrombocytopenia. Ind J Tuberc 1989; 36: 44-5

3. Flynn CT, Rainford DJ, Elinor Hope. Acute renal failure \& Rifampicin. Danger of unsuspected intermittent dosage. BMJ 1974;2: 482

4. Nessi $H$, Bonoldi GL, Redaelli B, Di Fillipo G. Acute renal failure after rifampicin : a case report \& survey of the literature. Nephron 1976; 16: $148-53$

5. Jerome Rossert. Drug induced acute intersticial nephritis. Kidney additional supporting evidence for Rifampicin induced AIN ${ }^{8}$. BUT OUR PATIENT HAD CONFIRM ED AIN (BIOPSY PROVEN) WITHOUTPERIPHERALBLOOD EOSINOPHILIA.

C. Oliguria is reported to be associated in $>90 \%$ cases of Rifampicin induced AIN at presentation and requires 2- 6 dialysis ${ }^{\circ}$. HOWEVER OUR CASE DID NOT HAVE OLIGURIA AND DID NOT RECEIVE DIALYSIS BUT RECOVERED FULLY WITH APPROPRIATE M ANAGEM ENT.

\section{Conclusions:}

Tuberculosis has to be ruled out in all cases of FUO. In the presence of non resolving pulmonary shadows especially involving upper lobes, even after treatment with adequate antibiotics, further evaluation for TB is essential. Investigations like BAL (Bronchoalveolar lavage) and AFB culture has to be used in FUO cases with radiological picture suggestive of TB. Serology for Tuberculosis and even sputum PCR may not be reliable investigations due to lack of standardisation.

Intersticial nephritis, although not as common as ATT induced Hepatitis in clinical practice, has to be considered in patients on ATT with acute renal failure even during maiden administration of ATT (Rifampicin). Early diagnosis of this complication and identifying, withdrawing the responsible drug is the key to prevent further deterioration. Early referral to a tertiary centre for evaluation of acute renal failure in patients on ATT is essential in further management of this potentially reversible complication.

international 2001;60:804-817

6. Kim MJ, Heim M , M ayr M . Nephrol Dial Transplant 2010; 25(5): 1716-9

7. Bassi L, Pernal G, Silvestri LG. Antibodies against rifampicin in patients with Tuberculosis after discontinuation of daily treatment. Am Rev Resp Dis 1976; 114: 1189-90

8. Latus J, Amann K, Braun N, Alscher MD, Kimmel M. Tubulo intersticial nephritis in active Tuberculosis- a single centre experience. Clin Nephrol 2012; 78(4): 297-302

9. Prakash J, Kumar NS, Saxena RK, Verma U. Acute renal failure complicating Rifampicin therapy. JAPI 2001; 49: 877-880 